\title{
Geographic variation in resilience: an experimental evaluation of four rocky intertidal assemblages
}

\author{
Letitia L. Conway-Cranos ${ }^{1,2, *}$ \\ ${ }^{1}$ Department of Ecology and Evolutionary Biology, University of California, Santa Cruz, California 95060, USA \\ ${ }^{2}$ Present address: Northwest Fisheries Science Center, Seattle, Washington 98112, USA
}

\begin{abstract}
Resilience is an increasingly important aspect of ecological theory and management, yet natural variation in resilience remains poorly understood. I quantified spatial variation in resilience by calculating recovery rates and trajectories in the rocky intertidal ecosystem across biogeographic regions along the coast of California in 4 intertidal assemblages dominated by taxa with contrasting dispersal distances, lifespans, and trophic positions. Here I have shown that resilience itself can vary dramatically across taxa and biogeographic regions and that this variation may be understood in the context of the life history characteristics, the ecology of the disturbed taxa, and the pathway by which recovery occurs. Overall, the barnacle and turf algal assemblages displayed the fastest recovery rates, while the mussel and rockweed assemblages showed recovery rates that were the slowest and most variable. Significant variation in both recovery rates and trajectories across regions in the invertebrate-dominated assemblages indicated that regional differences in the delivery of propagules were potential drivers in differences in recovery rates for these taxa. By contrast, regionally varying recovery trajectories, but not recovery rates, in the algal-dominated assemblages suggested difference in timing of key events driving the recovery process. The effect of disturbance magnitude on recovery rates was consistent across regions for the mussel and rockweed assemblages, but in the barnacle and turf algal assemblages, the effect of disturbance size differed across regions, indicating variability in processes that drive edge effects. In all 4 assemblages, geographic differences in recovery trajectories outweighed differences across disturbance sizes.
\end{abstract}

KEY WORDS: Resilience · Recovery · Succession · Intertidal $\cdot$ Chthamalus $\cdot$ Mytilus $\cdot$ Endocladia Silvetia Resale or republication not permitted without written consent of the publisher

\section{INTRODUCTION}

Understanding the manner in which communities respond to perturbations is one of the most fundamental goals of ecology (Connell \& Slatyer 1977, Bazzaz 1979). Because both natural and anthropogenic disturbances can result in loss of biomass, understanding the process of recovery after a disturbance is both an important component of understanding how communities function as well effectively managing natural resources. The rate of recovery after such perturbations can be thought of as 'resilience' (Con- nell \& Sousa 1983), and while there has been much theoretical (e.g. Holling 1973) and practical (e.g. Leslie \& Kingzig 2007) attention to this concept, experimental explorations of resilience are rare (but see Allison 2004, Viejo 2009). Resilience is often treated as a feature of ecosystems that is desirable from a management perspective (e.g. Elmqvist et al. 2003) and relatively static in the absence of human perturbations (e.g. van Nes \& Scheffer 2007), a perspective that overlooks the potential importance of natural variation in resilience. Such variation will be driven in part by the life history characteristics of the 
organisms present at a given location (e.g. lifespan and dispersal distance) and in part by location-specific factors that govern the delivery and survival of new propagules, resulting in a geographically varying mosaic of underlying recovery potential. As such, the recovery potential of a given location is thus likely to arise from both species-specific qualities and geographically or spatially varying ones.

Ecological recovery from natural or anthropogenic disturbances can be understood in (at least) 2 complementary ways. First, the timeframe of convergence with an undisturbed condition can be estimated by determining the 'recovery rate', or the amount of time it takes for ecological recovery to occur. Recovery itself can be defined as a statistical value expressed as some a priori similarity to the undisturbed condition, which also may change over time. Second, recovery can be thought of as an 'ecological trajectory', the temporal progress of ecological states prior to defined recovery. This can be considered the ecological path taken between disturbance and recovery. Differences in such paths reflect varying contributions and timing of different ecological mechanisms directing the successional process. While recent studies have improved our understanding of recovery and the successional mechanisms that can govern this process (e.g. Crain et al. 2008, Lebrija-Trejos et al. 2010), our understanding of the extent to which recovery rates and trajectories may vary across taxa and spatial locations remains limited (but see Kinnetic Laboratories 1992, Bertness et al. 2002, Petraitis \& Dudgeon 2005).

Empirical work in both terrestrial (e.g. Phillips \& Shure 1990, Hubbell et al. 1999, Dietze \& Clark 2008) and marine (e.g. Paine \& Levin 1981, Farrell 1991, Petraitis \& Dudgeon 1999, Cervin et al. 2004) systems has shown that community processes and rates of recovery differ among disturbed patches of different sizes, often in seemingly inconsistent ways. In part, this is because the surrounding edge habitat may have strong and varying effects on new recruits. If organisms in intact edge habitat have a negative impact on incoming propagules or early life history stages, large disturbances may be expected to recover more quickly than small ones, since the center of such disturbances would represent a refuge from negative edge effects. Negative edge effects can be the result of shading by intact adjacent canopy species (Bazzaz 1979), from algal whiplash (Jenkins et al. 1999, Cervin et al. 2004), predation of larvae by adults (Young \& Gotelli 1988), or grazing by herbivores that take refuge in undisturbed habitat (Sousa 1984). Conversely, surrounding biota may have a positive effect on recovering propagules through amelioration of physiological conditions (Bertness \& Shumway 1993) or gregarious settlement (Raimondi 1990). In such cases, smaller disturbances would be expected to exhibit faster recovery rates than large ones. Thus, insights can be obtained into the processes responsible for driving variation in recovery rates and trajectories by examining community response to disturbances of varying sizes. Typically, studies on the effects of disturbance size on recovery use a small number (2 to 3 ) of contrasting patch sizes (Sousa 1984, Farrell 1989, Bertness et al. 2002), which permits comparing dynamics between different disturbance sizes but does not permit characterizing recovery with respect to disturbance size. Knowing the shape of the relationship between disturbance size and recovery rate is consequently better addressed if a gradient of potential disturbance sizes is examined.

There is growing awareness among ecologists of the importance of understanding the extent to which community dynamics are influenced by factors that vary predictably with spatial or geographic location (Underwood 2000, Menge et al. 2004, Coleman et al. 2006, Burrows et al. 2009). However, much of our current understanding of many important ecological processes come from experiments conducted within relatively limited spatial scales (e.g. Paine 1966, Tilman 1985). Those studies that have investigated succession across more broad geographic scales in both marine (e.g. Chapman \& Underwood 1998, Dudgeon \& Petraitis 2001, Foster et al. 2003) and terrestrial (e.g. Dietze \& Clark 2008) systems have demonstrated that the recovery process can vary dramatically in space and that the relative importance of the mechanisms driving recovery can change over spatial scales in response to geographic variation in propagule availability, consumer pressure, abiotic factors, or some combination thereof. However, because of the data-intensive nature of studying rules of assembly, studies examining recovery or succession often sacrifice a broad-scale approach for a more detailed understanding of succession at a location or within a particular type of ecological community. In marine systems, there may be significant variation in recovery rates and trajectories in response to variation in larval supply, nutrient delivery via upwelling, temperature, or variation in consumer pressure (Connolly et al. 2001, Menge et al. 2004, Coleman et al. 2006, Broitman et al. 2008). These location-specific suites of factors are likely to favor particular traits of recovering species. Because many intertidal species occur throughout much of the west coast of the USA, 
despite dramatic abiotic and biotic differences across this range (Blanchette et al. 2008), the California current ecosystem provides a unique opportunity to examine recovery rates across a common suite of species, yet displays meaningful variation in abiotic and biotic factors that could lead to different recovery dynamics in different locations. While there is agreement that processes that vary on broad spatial scales are likely to be important in determining the outcome of succession (e.g. Chapman \& Underwood 1998, Dudgeon \& Petraitis 2001, Foster et al. 2003), this possibility has been rarely explored experimentally across multiple intertidal assemblages (but see Kinnetic Laboratories 1992). Here, I demonstrate a pattern of community-specific spatial variation in ecological resilience by quantifying recovery rates and trajectories in 4 different intertidal assemblages across a broad spatial scale on the west coast of the United States.

\section{Background: study system}

The west coast of the USA has been remarkably well characterized with respect to geographic patterns in water temperature, recruitment (e.g. Broitman et al. 2008), productivity (Connolly et al. 2001, Menge et al. 2004), species interactions (Menge et al. 2004), and intertidal biota (Blanchette et al. 2008). Many of these studies have highlighted Point Conception (Santa Barbara County, California) as a major feature of this coast that delineates a boundary between warm and cool water masses (Hickey 1979, 1993, Cudaback et al. 2005), biogeographic regions (Blanchette et al. 2008), and upwelling regimes (Menge et al. 2004). In addition to these abiotic characteristics, Broitman et al. (2008) observed coastwide variation in recruitment dynamics in the mussel zone for intertidal mussels and 2 species of barnacles, finding low levels from northern California to Point Conception, with higher levels of Balanus recruitment and low levels of Mytilus recruitment south of Point Conception. The other species of barnacle, Chthamalus, showed more variability, with the highest recruitment rates observed in the Santa Barbara Channel (south of Point Conception) and variable but generally lower recruitment north of Point Conception (Broitman et al. 2008).

A broad-scale investigation of recovery of much bigger experimental clearings in the mussel Mytilus californianus and the turf alga Endocladia muricata assemblages has also been conducted along the California coast by Kinnetic Laboratories (1992) and Fos- ter et al. (2003). While the geographic range of this study was set north of the present study and did not span Point Conception, there is spatial overlap with the present study at 2 sites: Pt. Sierra Nevada (see Fig. 1) and Diablo Canyon, a site approximately $60 \mathrm{~km}$ north of one of the current study sites (Stairs, see Fig. 1) (Kinnetic Laboratories 1992, Foster et al. 2003). The mussel component of these studies revealed slow recovery rates at all sites, with no significant relationship with latitude or season of clearing, and found slightly higher recovery rates at Diablo Canyon than at Pt. Sierra Nevada (Kinnetic Laboratories 1992). The Endocladia component of the study found different geographic patterns in recovery rate that depended on the season in which the disturbances were made, with the highest rates observed in spring-cleared plots at a site north of San Francisco and the lowest recovery rates observed at Diablo Canyon in fall-cleared plots (Foster et al. 2003).

\section{Background: intertidal assemblages}

The rocky intertidal is an ideal place to conduct a broad-scale investigation of resilience since ecologically relevant experimental disturbances can be made on spatial scales on the order of meters rather than kilometers and can be observed over the course of a few years rather than 100s of years, as would need to be the case for many other ecosystems. Because of the characteristic immersion gradient in rocky intertidal habitats, different species dominate at different tide heights (Menge \& Branch 2001), permitting the exploration of recovery dynamics across zones consisting of species that display variation in life history characteristics (e.g. lifespan and dispersal capability) over spatial scales that are experimentally tractable.

I chose 4 intertidal zones characterized by different taxa that are common components of the rocky ecosystem across the west coast of North America (Blanchette et al. 2008) and because the dominant taxa in each assemblage exhibit variation in life history characteristics, such as lifespan and dispersal capability, that could lead to fundamentally different recovery dynamics. These taxa are: (1) the acorn barnacle Chthamalus dalli/fissus (hereafter, Chthamalus), a short-lived (1 to $3 \mathrm{yr}$ ) filter-feeder that has pelagic larvae, occurs as a distinct band in the high intertidal, and may disperse 10s to 100s of kilometers (Newman \& Abbott 1980, Shanks et al. 2003); (2) the filter-feeding mussel Mytilus californianus (hereafter, Mytilus), which is longer-lived ( 7 to $20 \mathrm{yr}$ ), 
occurs in the low-mid intertidal and also has pelagic larvae with dispersal potential similar to Chthamalus (Haderlie \& Abbott 1980, Suchanek 1981); (3) the rockweed Silvetia compressa (hereafter, Silvetia), an alga which disperses centimeters to meters, occurs in the mid intertidal and can live for up to $7 \mathrm{yr}$ (Gunnill 1980, Brawley \& Johnson 1991, Hays 2006); and (4) the red turf alga Endocladia muricata (hereafter, Endocladia), which typically occurs in the high-mid intertidal and is not well-studied with respect to either dispersal capability or lifespan but, as a red alga, likely has a shorter dispersal capability than Chthamalus or Mytilus and a shorter lifespan than Silvetia.

I predicted that I would observe faster recovery of the shorter-lived taxa (Chthamalus and Endocladia) and that recovery rates of Mytilus and Chthamalus would be reflective of previously observed geographic patterns in recruitment (Broitman et al. 2008) and growth (Blanchette et al. 2007), with highest recovery rates south of Point Conception.

\section{MATERIALS AND METHODS}

\section{Study location}

To determine the extent of geographic variation in recovery dynamics along the west coast of the United States, I selected 3 rocky intertidal sites along the California coast that spanned 2 biogeographic regions: Point Sierra Nevada (north of Point Conception), Stairs (near Point Conception), and Point Fermin (south of Point Conception) (Fig. 1).

\section{Experimental disturbances and censuses}

I took a regression approach in understanding the effects of disturbance size on recovery rates and trajectories. As such I selected a gradient of disturbance sizes to implement experimentally. This permits a more precise understanding of the relationship between disturbance size and recovery dynamics and allows using disturbance magnitude as a meaningful continuous covariate in statistical analyses. For logistical and ethical reasons, individual disturbance sizes in the gradient were not replicated within sites and assemblages. While this lack of replication places limitations on the type of statistical test that may be used to compare dynamics across the treatments, it is nonetheless appropriate for a regression design.
In Fall of 2003, I established 8 recovery plots of the following sizes: $8 \times 12 \mathrm{~cm}, 10 \times 15 \mathrm{~cm}, 13 \times 20 \mathrm{~cm}, 16$ $\times 24 \mathrm{~cm}, 22 \times 33 \mathrm{~cm}, 30 \times 45 \mathrm{~cm}, 40 \times 60 \mathrm{~cm}$, and $50 \times$ $75 \mathrm{~cm}$ in the Chthamalus, Mytilus, Endocladia, and Silvetia communities at each of the 3 study locations. These clearings were chosen to represent a suite of disturbance sizes that fell within the natural range for this system (e.g. Sousa 1984). The size of disturbance could potentially be caused by wave dislodgement, sand scour, or tar smothering. In each intertidal zone, plots were haphazardly located within the tidal elevation dominated by the target species (Chthamalus, Mytilus, Endocladia, or Silvetia), avoiding vertical walls, large crevices, and tidepools. I also established three $50 \times 75 \mathrm{~cm}$ control (undisturbed) plots interspersed among the disturbed plots in each assemblage. In the disturbed (recovery) plots, I cleared the substratum of all visible biota and then burned each plot with a hand-held propane torch to remove microscopic propagules (sensu Lubchenco \& Menge 1978, Murray \& Littler 1978, Sousa 1979, Foster et al. 2003). All experimental clearings were made within a matrix of intact biota. Sampling consisted of placing $2.54 \mathrm{~cm}$ diameter $\left(1 / 4^{\prime}\right)$ PVC rectangular frames (for larger plots) or thin stainless steel rectangular tubes (for smaller plots) of the appropriate size strung with a uniform grid of monofilament line and noting the identity and location in the grid of species occupying the primary substratum and canopy (if present) under each intersection; thus for a given point, multiple species could be recorded. If it lay within the experimental plot, canopy-forming algae from sur-

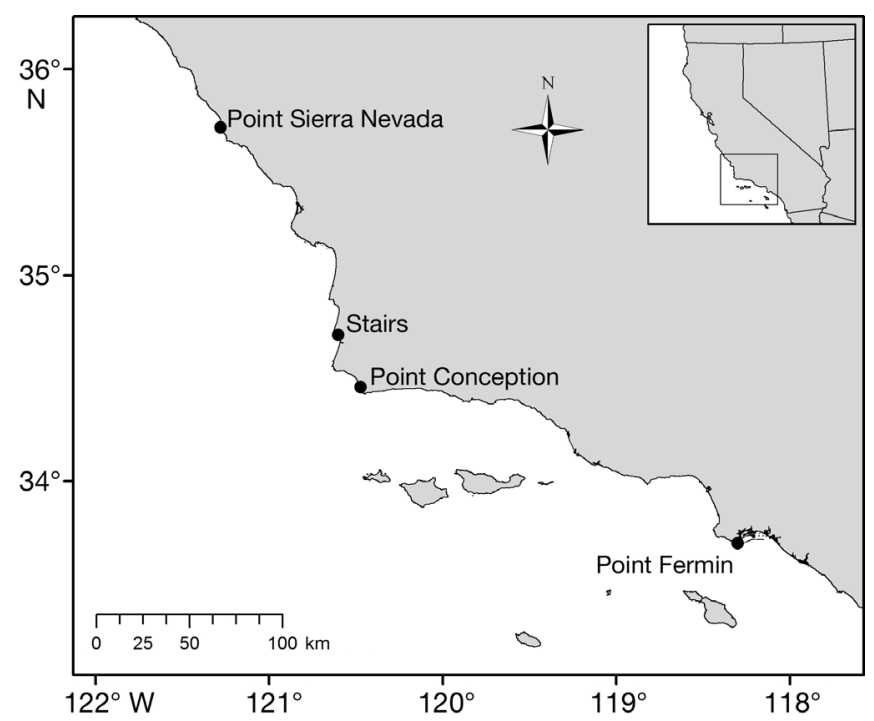

Fig. 1. Study locations north of (Point Sierra Nevada), near to (Stairs), and south of (Point Fermin) Point Conception in California, USA 
rounding undisturbed plots were counted as being part of the plot. Due to the range of plot sizes, plots differed in the number of points used to sample them, such that the largest 5 plots were sampled with 100 evenly spaced points, and the 3 smallest plots were sampled with 50, 40, and 30 points, respectively. All plots were marked with stainless steel screws in the corners to ensure consistency in the placement of the quadrat on each sampling visit. Plots were sampled prior to clearing and reassessed every 4 to 6 mo until spring of 2008, a span of $52 \mathrm{mo}$, encompassing 11 censuses.

\section{Measuring recovery rates in the Mytilus, Chthamalus and Endocladia assemblages}

Recovery was determined by quantitatively comparing each disturbed plot to undisturbed control plots by calculating the Bray-Curtis similarity index (Bray \& Curtis 1957) between every cleared plot and each of the 3 control plots within that intertidal assemblage, resulting in 3 estimates of community similarity for each recovery plot at each time interval. I also calculated the similarity of the controls to one another (sensu Murray \& Littler 1978, Kinnetic Laboratories 1992, Foster et al. 2003) to establish a recovery 'threshold' that was defined as the point in time at which the mean similarity of a disturbance plot became greater than or equal to the mean similarity of the controls to one another $(n=3)$ minus 1 standard deviation. In this way, natural variability at each site in each intertidal assemblage helped to define the recovery threshold. Once a plot passed the threshold, it was considered recovered regardless of later fluctuations. To estimate recovery rate for every disturbance plot, I calculated the slope of the line passing through 2 points i.e. the point at which the plot was disturbed and the point in time at which it recovered such that for a given plot, $x$-values were months since disturbance and $y$-values were BrayCurtis similarity to each control plot. For plots that had not met the recovery threshold, rate of recovery was calculated using the 52 mo of available data.

\section{Measuring recovery rate in the Silvetia assemblage}

In the Silvetia assemblage, a different metric for measuring recovery rate was used due to the high percent of algal canopy and bare rock in both recovery and control plots as well as the spatial and temporal variability of canopy cover. Here I simply used the cover of Silvetia in the control plots as the metric for recovery, calculating the mean percent cover (across the 3 control plots) minus 1 standard deviation to define the threshold for recovery compared to the percent cover of Silvetia in each recovery plot. As with the other assemblages, I estimated recovery rate by calculating the linear slope using only the time interval immediately after clearing and the interval at which the percent cover reached threshold level. Since Silvetia exhibited greater seasonal fluctuations compared with other assemblages, each plot had to remain in this recovery envelope over at least 2 consecutive seasons (e.g. both fall and spring samplings) to be considered recovered.

\section{Comparing recovery rates}

To assess differences in resilience across assemblages, I compared the distributions of the observed times to recovery. The maximum possible observed recovery time was 52 mo (the end of the study). To determine the effects of plot size and geographic location (site), I conducted an analysis of covariance (ANCOVA) (Systat v.12) within each assemblage with recovery rate as the dependent variable, geographic location as the main predictor variable, and disturbance size (the natural log of the plot area) as the covariate. This analysis was chosen over a nested ANOVA because it permits the explicit inclusion of a continuous covariate (disturbance size). When there was a non-significant interaction term, I removed this term and applied the reduced model. I applied Tukey's post-hoc test to compare pairs of sites when appropriate. To further examine the relationship between disturbance size and recovery rate within sites, I conducted linear regressions (Systat v.12) using recovery rate as the dependent variable and disturbance size as the predictor variable.

\section{Treatment of outliers and natural catastrophes}

There were 2 plots with recovery rates that were drastically different from the other plots, and inclusion of these plots in the analyses masked underlying patterns among sites and among disturbance sizes. These plots both had a priori reasons related to their placement that could have led to the anomalous results. The Endocladia plot at Stairs was located in a more offshore and somewhat lower tidal elevation than the other plots, which could have led to its being more subject to sand and wave scour than appeared 
to be typical for the rest of the assemblage, thus resulting in its slower observed recovery rate. The mussel plot at Stairs was located in a depression in the substratum such that the vertical thickness of the mussel bed surrounding this plot was greater than the rest of the plots and, as such, likely contributed to its much faster recovery by near-immediate collapse of the adjacent mussel bed into the clearing. These plots were included in Fig. 6 (labeled with asterisks) but are treated as outliers and omitted from all statistical analyses, averaged figures, and regression lines. At the conclusion of the study (spring of 2008), 2 of the Endocladia plots at Stairs were obliterated by breaking rock. These plots had already recovered so this occurrence did not affect the analysis of recovery rates but since the analysis of trajectories included all sample intervals (described below), I left this interval out of the comparison of trajectories for the Endocladia assemblage so as not to violate the statistical analysis, which requires no missing data.

\section{Measuring community recovery trajectories within assemblages}

Analysis of recovery trajectories permits explicitly comparing the timing and nature of ecologically relevant shifts in community composition across disturbance sizes and geographic locations. To compare community recovery trajectories, for each sample interval I used a method that is analogous to a 2nd stage MDS (PRIMER v.6). This technique is described in Clarke et al. (2006) whereby community change over time in a given plot can be first represented using a similarity matrix (1st stage MDS) followed by an explicit comparison of multiple similarity matrices representing different plots (2nd stage MDS), permitting visual inspection of recovery trajectories in MDS space and comparing them statistically using ANOSIM (PRIMER, v.6). However, this powerful technique has 2 drawbacks: (1) it does not readily integrate undisturbed or reference plots into graphical depictions of recovery trajectories, and (2) It is not possible to conduct a 2nd stage analogue to SIMPER (PRIMER v.6) whereby important time intervals and taxa may be identified as driving observed differences between trajectories.

To compare community recovery trajectories in a way that both incorporates the relationship between experimental and reference plots and that also permits the determination of the causes of observed differences in recovery trajectories, I first calculated the difference between species-specific cover in each disturbed plot and the mean cover for each species in the control plots, thus integrating the relationship between recovery and control plots into the analysis. I then used non-normalized principal component analysis (PCA) to reduce these differences in species composition at each timestep to a single non-normalized PCA axis or factor (PC1), calculating the coefficients for each species in the community (PRIMER v.6), similar to the approach taken by O'Leary \& McClanahan (2010) who used principle component factors for a multiple regression analysis and then related coefficients to particular taxa. Loadings for each species were calculated by finding the Pearson correlation between the composite variable (PC1) and the original data (Systat v.12). I compared trajectories by calculating Euclidean distances of this single principal component between all pairs of recovery plots within a species assemblage over time and across locations. I then used MDS on the Euclidean distances to compare trajectories across disturbance sizes and across locations (PRIMER v.6). To assess community changes over time in the undisturbed (control) plots, I randomly chose 2 of the 3 control plots and calculated the principal component score on the difference between them, generating one control value for every timestep at each site. I included these control trajectories in graphical depictions of recovery (MDS plots, PRIMER v.6) but did not include them in statistical comparisons of recovery trajectories. I conducted 1-way ANOSIM tests (PRIMER v.6) to assess whether recovery trajectories differed across geographic locations and another set of 1-way ANOSIM tests to assess whether trajectories differed with respect to disturbance size.

To determine which time intervals and which taxa drove observed differences in trajectories, I conducted SIMPER analyses (PRIMER v.6), allowing me to determine the important time intervals driving differences in recovery trajectories, as well as the taxa contributing to these differences, through examination of the PC1 coefficients. In this way it was possible to identify not only when trajectories differed but also which taxa were likely responsible for the observed differences. However, since plots were sampled every 4 to $6 \mathrm{mo}$, it is possible that differences in timing of important recovery events across sites or disturbances were treated as a single event and thus obscured. For this reason, the analysis is conservative such that only events that occur at timescales greater than the sampling frequency are detected. 


\section{RESULTS}

\section{Recovery rates}

Although there was considerable variation in the amount of time to recovery, the median recovery times for Chthamalus and Endocladia communities were 15 and $25 \mathrm{mo}$, respectively, while those observed in the Mytilus and Silvetia communities were much longer, having median recovery times of 40 and 35 mo, respectively. Indeed, in both the Mytilus and Silvetia assemblages, $25 \%$ of the plots displayed recovery times that were greater than or equal to the length of the study (52 mo) (Fig. 2). Despite this, unrecovered plots generally exhibited temporal trends toward convergence with control plots (Figs. $3 \& 4$ ). Both invertebrate assemblages (Chthamalus and Mytilus) showed the slowest recovery at Stairs (near Point Conception) (Table 1; Fig. 5), and the highest recovery rates for Chthamalus were observed south of Point Conception. By contrast, the algal assemblages showed more variability with respect to across-site variation in recovery rates such that recovery rates across sites were statistically similar for both the Endocladia and Silvetia assemblages, although there was a clear trend towards slower recovery at the northern site (Point Sierra Nevada) in the Silvetia assemblage (Table 1; Fig. 5).

The effect of disturbance size on recovery rate was most consistent across the 3 study locations in the Mytilus assemblage, where recovery rates slowed with increasing plot sizes at all 3 sites (Fig. 6). In the Chthamalus assemblage, the negative relationship between disturbance size and recovery rate was sig-

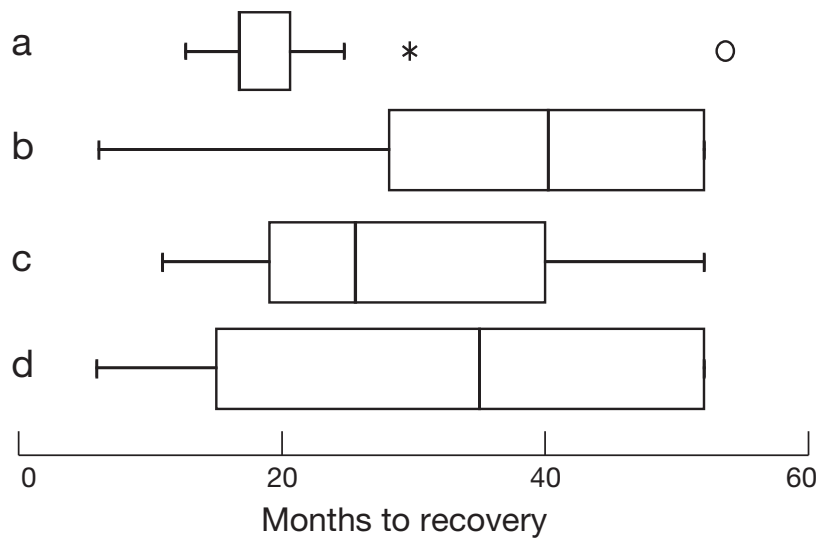

Fig. 2. Distribution of observed recovery times for all plots in the (a) Chthamalus, (b) Mytilus, (c) Endocladia, and (d) Silvetia assemblages at each of the 3 study locations over the course of the 52 mo study. The asterisk indicates values $>1.5$ times the interquartile range, and the open circle indicates values $>3$ times the interquartile range nificant only at Stairs, and there was no relationship at any site for the Silvetia assemblage (Fig. 6). By contrast, Endocladia showed a positive relationship between clearing size and recovery at Stairs and a trend towards a negative relationship at Point Sierra Nevada (Fig. 6), resulting in a significant site by disturbance area interaction (Table 1).

\section{Recovery trajectories}

The PC1 axis in the Chthamalus assemblage explained $98 \%$ of the variation and so provided a highly robust metric for examining recovery trajectories over time. The community components with the highest loadings were bare rock and Chthamalus. Bare rock showed a strongly negative coefficient while both live and dead Chthamalus showed positive coefficients (Table 2), signifying that a disturbed plot would transition from negative PC1 scores (more rock relative to controls) to positive ones (more Chthamalus) over time. An MDS plot comparing trajectories revealed that Chthamalus community recovery trajectories at Stairs and Point Fermin were the most different and that all 3 sites had recovery trajectories that were distinct from one another (Fig. 7). An ANOSIM test showed that there was a significant site effect $(\mathrm{R}=0.49 ; \mathrm{p}=0.001)$ and no effect of plot size $(R=-0.183 ; p=0.986)$, suggesting that site-specific differences in recovery trajectories were more important than the dynamics of individual plots of varying size.

Analysis of the timing of community shifts in recovery showed different patterns across the 3 study sites. No timesteps stood out as contributing to the majority of the variation between observed recovery trajectories at Point Sierra Nevada and Stairs (Table 3). By contrast, the 3 intervals driving the observed differences between recovery trajectories observed at Point Sierra Nevada and Point Fermin were all in the year 2005 (Table 3). At these timesteps, Point Fermin had more positive PC1 values, indicating that there were major shifts toward communities consisting of Chthamalus and less bare rock relative to control plots than at Point Sierra Nevada. Similarly, 3 time intervals drove nearly $40 \%$ of the variation in observed differences in community recovery trajectories between Stairs and Point Fermin in the Chthamalus assemblage (Table 3 ) however these intervals were both early ( 0 and $3 \mathrm{mo}$ ) and later in the successional process $(19 \mathrm{mo})$. The differences appeared to be driven by increased rock in the recovery plots relative to the controls (a more negative average PC1 

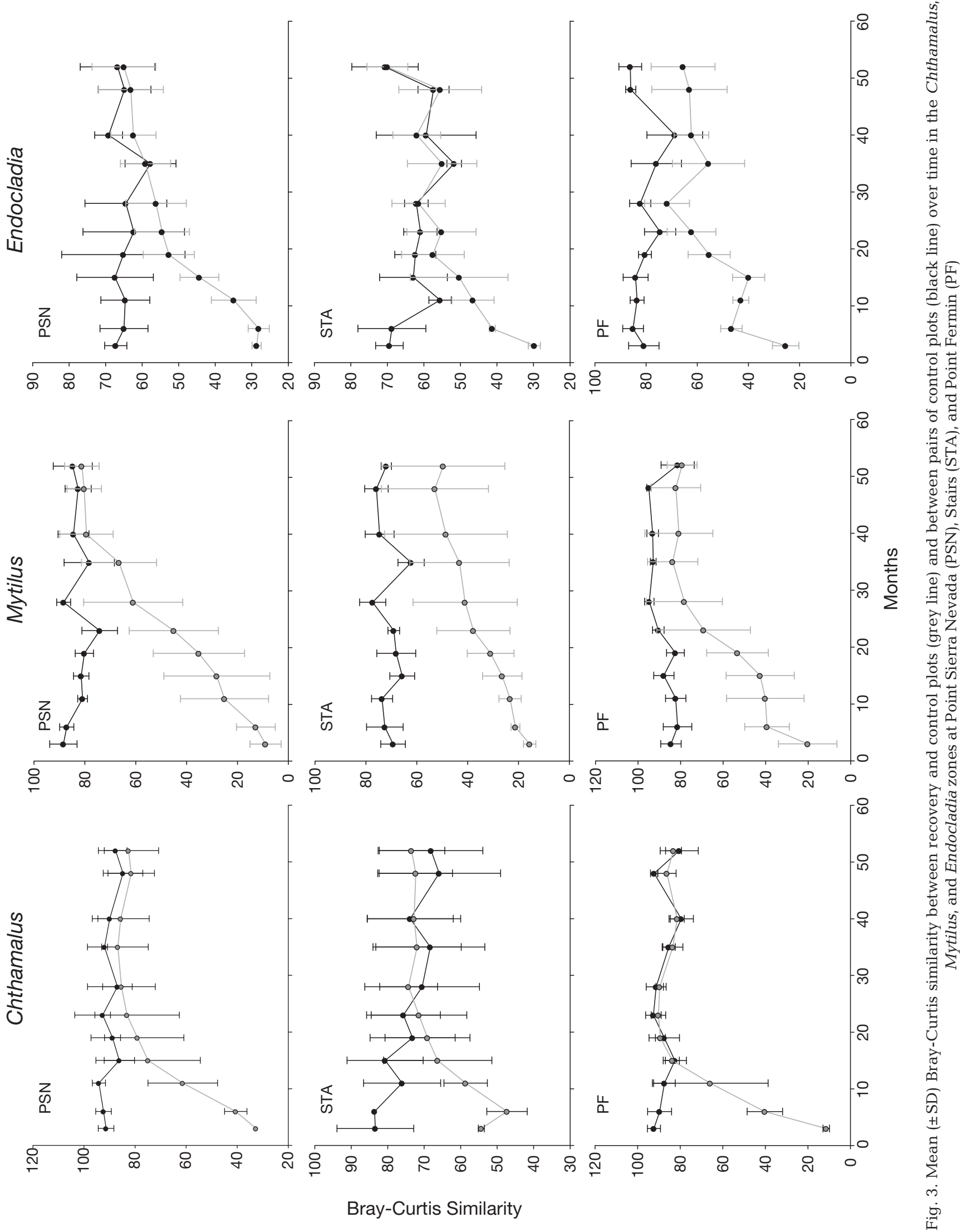

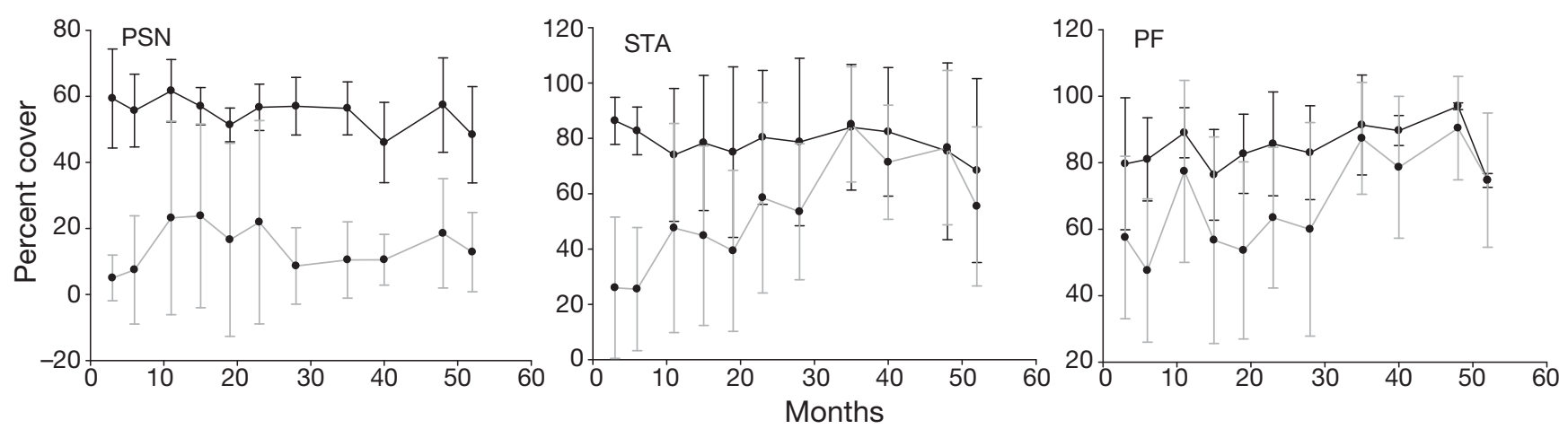

Fig. 4. Mean $( \pm \mathrm{SD})$ percent cover of Silvetia in recovery plots (grey line) and and control plots (black line) over time in the Silvetia zone at Point Sierra Nevada (PSN), Stairs (STA), and Point Fermin (PF)

Table 1. Analyses of covariance using recovery rate as the dependent variable, geographic location (site) as the factor, and disturbance area (size) as the covariate

\begin{tabular}{|lcccc|}
\hline Source & df & MS & $F$ & $\mathrm{p}$ \\
\hline Chthamalus & & & & \\
Site $\times$ Size & 2.00 & 1.38 & 0.93 & 0.41 \\
Site & 2.00 & 48.74 & 33.16 & 0.00 \\
Size & 1.00 & 0.76 & 0.51 & 0.48 \\
Mytilus & & & & \\
Site $\times$ Size & 2.00 & 0.66 & 3.07 & 0.07 \\
Site & 2.00 & 3.69 & 14.16 & 0.00 \\
Size & 1.00 & 11.94 & 45.85 & 0.00 \\
Endocladia & & & & \\
Site $\times$ Size & 2.00 & 3.04 & 5.78 & 0.01 \\
Site & - & - & - & - \\
Size & - & - & - & - \\
Silvetia & & & & \\
Site $\times$ Size & 2.00 & 4.79 & 0.41 & 0.67 \\
Site & 2.00 & 29.94 & 2.75 & 0.09 \\
Size & 1.00 & 30.77 & 2.82 & 0.11 \\
\hline
\end{tabular}

value) at Point Fermin at the 0 and 3 mo intervals followed by a shift to more Chthamalus in the recovery plots at Point Fermin than at Stairs at the 19 mo time interval (Table 3).
In the Mytilus assemblage, the PC1 axis described $75 \%$ of the variation in community composition. The species with the highest loadings and positive coefficients were Mytilus californianus and the gooseneck barnacle Pollicipes polymerus, while bare rock had highly negative coefficients (Table 2), indicating that a recovering plot would transition from more negative scores (more rock relative to the controls) towards more positive ones (a community comprised mainly of Mytilus and Pollicipes). Mytilus community recovery trajectories differed significantly across geographic locations $(\mathrm{R}=0.24, \mathrm{p}=0.003)$ but not by disturbance size ( $R=0.07, p=0.238$ ) (Fig. 7). SIMPER analysis showed that between Point Sierra Nevada and Stairs, the greatest differences were later in succession (Table 3), with these differences contributing to nearly half of the observed variation. PC1 values were higher (more positive) at Point Sierra than Stairs at all 3 intervals, indicating a shift in community composition towards recovery (higher cover of Mytilus and Pollicipes) at Point Sierra Nevada but not at Stairs at these timesteps. By contrast, the interval driving the majority of variation in recovery trajectories between Point Sierra Nevada and Point Fermin was somewhat earlier in succession, such that Fall of 2005 (23 mo)
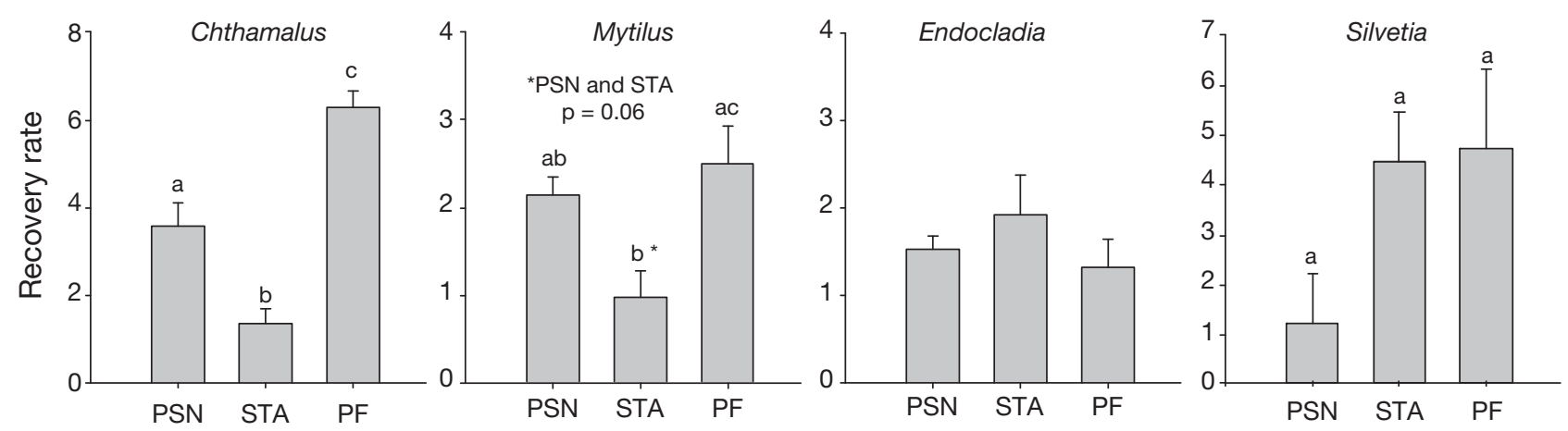

Fig. 5. Average recovery rates (across disturbance sizes) across sites within the Chthamalus, Mytilus, Endocladia, and Silvetia assemblages. Different letters indicate significant $(\mathrm{p}<0.05)$ differences. Data are shown as mean $( \pm$ SEM) 


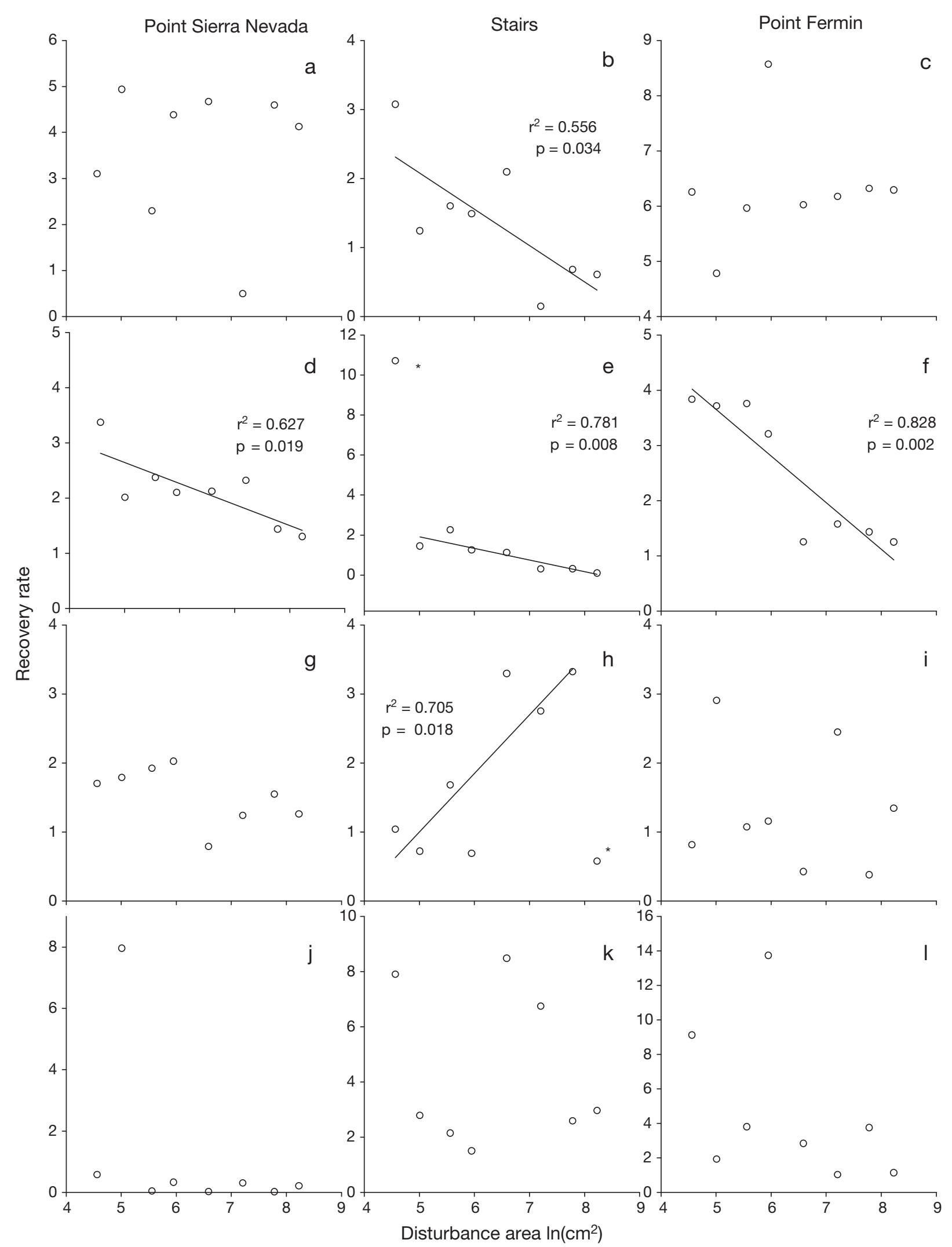

Fig. 6. Linear regressions between recovery rate and disturbance size within each assemblage at each location for the (a-c) Chthamalus, (d-f) Mytilus, (g-i) Endocladia, and (j-l) Silvetia assemblages. Asterisks indicate outliers that were not used in the calculation of the regression statistics or line 
Table 2. Coefficients and loadings for each species from the principal components analysis (non-normalized). Data shown for species with loadings with an absolute value above 0.25

\begin{tabular}{|c|c|c|}
\hline $\begin{array}{l}\text { Assemblage } \\
\text { Species }\end{array}$ & $\begin{array}{c}\text { Latent } \\
\text { eigenvector } \\
\text { (coefficient) }\end{array}$ & $\begin{array}{c}\text { Loading } \\
\text { (absolute } \\
\text { value) }\end{array}$ \\
\hline \multicolumn{3}{|l|}{ Chthamalus } \\
\hline Chthamalus dalli/fissus & 0.69 & 0.99 \\
\hline Bare rock & -0.72 & 0.99 \\
\hline Dead Chthamalus spp. & 0.03 & 0.33 \\
\hline \multicolumn{3}{|l|}{ Mytilus } \\
\hline Mytilus californianus & 0.75 & 0.97 \\
\hline Bare rock & -0.66 & 0.95 \\
\hline Pollicipes polymerus & 0.03 & 0.37 \\
\hline Lottia scabra & -0.01 & 0.31 \\
\hline Dead Mytilus & 0.01 & 0.27 \\
\hline Dodecacaria fewkesi & 0.01 & 0.26 \\
\hline \multicolumn{3}{|l|}{ Endocladia } \\
\hline Endocladia muricata & -0.75 & 0.92 \\
\hline Bare rock & 0.63 & 0.87 \\
\hline Mastocarpus papillatus & -0.18 & 0.46 \\
\hline Brown crust (Ralfsiaceae) & 0.00 & 0.36 \\
\hline Cladophora columbiana & -0.06 & 0.28 \\
\hline $\begin{array}{l}\text { 'Petrocelis' phase of } \\
\text { Mastocarpus spp. }\end{array}$ & -0.03 & 0.26 \\
\hline $\begin{array}{l}\text { Mytilus galloprovincialis/ } \\
\text { trossulus }\end{array}$ & 0.00 & 0.26 \\
\hline \multicolumn{3}{|l|}{ Silvetia } \\
\hline Silvetia compressa & 0.82 & 0.90 \\
\hline Bare rock & -0.52 & 0.70 \\
\hline Mytilus californianus & 0.12 & 0.50 \\
\hline Cladophora columbiana & 0.03 & 0.41 \\
\hline Pollicipes polymerus & 0.01 & 0.37 \\
\hline $\begin{array}{l}\text { 'Petrocelis' phase of } \\
\text { Mastocarpus spp. }\end{array}$ & 0.07 & 0.34 \\
\hline Ralfsia spp. & 0.17 & 0.32 \\
\hline Tetraclita rubescens & 0.01 & 0.29 \\
\hline
\end{tabular}

contributed the most variation (Table 3). In this case, the PC1 score of Point Sierra Nevada was more negative relative to the highly positive value at Point Fermin, indicating that at this time interval, there were more early successional community components at Point Sierra Nevada and more late successional components at Point Fermin relative to the control plots (Table 3). The time intervals driving differences be-

Fig. 7. MDS plots of recovery trajectories in the Chthamalus, Mytilus, Endocladia and Silvetia assemblages. Each point represents the trajectory (change in PC1 score of the difference in species composition between control and recovery plots) over time for a given plot where numbers correspond to plot sizes such that $1=$ smallest and $8=$ largest. The trajectories of the difference between 2 random control plots for each assemblage at each site are depicted by the letter C. See Fig. 3 for site abbreviations
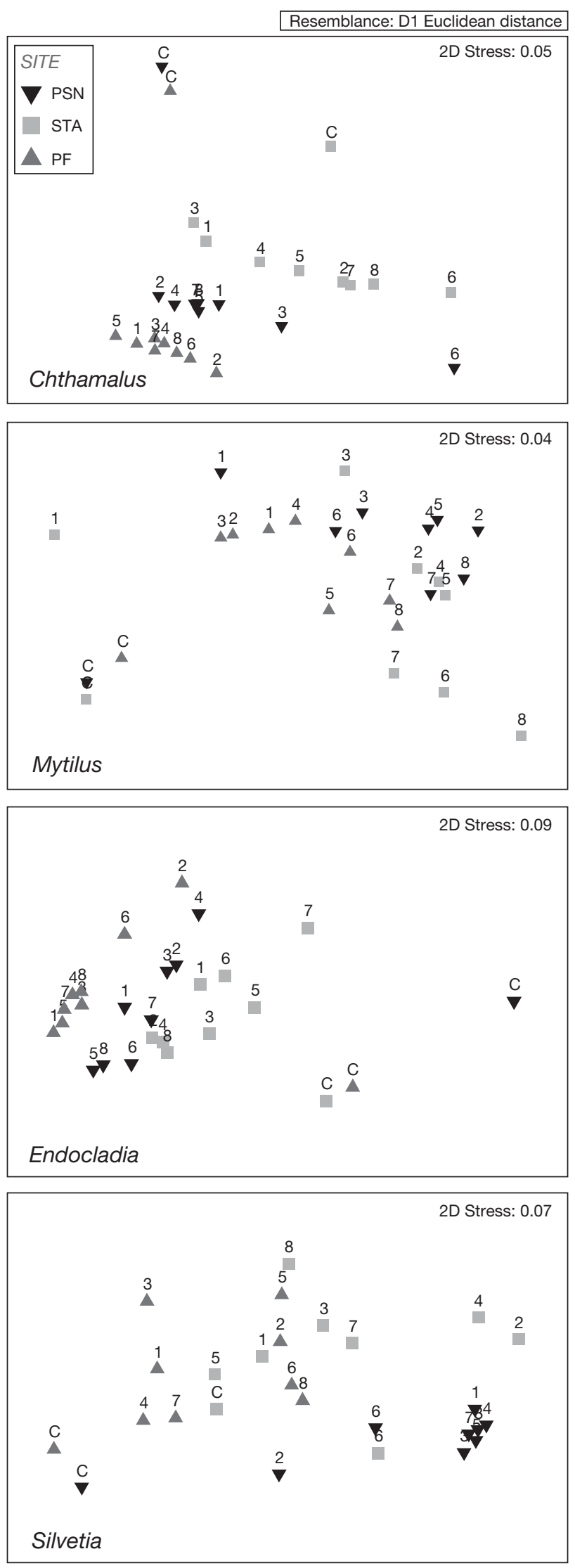
Table 3. Results of SIMPER analyses identifying the time intervals most responsible for driving observed differences between recovery trajectories across geographic locations. The intervals with the 3 highest percentage contributions to differences are presented for each pair of sites within each assemblage. The average PC1 scores at each site at the important intervals are also reported. (PSN, Point Sierra Nevada; STA, Stairs; PF, Point Fermin). Intervals are indicated by season (FA: fall; WI: winter; SP: spring; SU: summer) and year of disturbance, with months since disturbance (0 to 52) in parentheses

\begin{tabular}{|c|c|c|c|c|c|}
\hline \multirow{2}{*}{$\begin{array}{l}\text { Assemblage } \\
\text { Comparison }\end{array}$} & \multirow{2}{*}{$\begin{array}{l}\text { Interval } \\
\text { (months) }\end{array}$} & \multicolumn{3}{|c|}{ - Avg. PC1 } & \multirow[t]{2}{*}{ Percentage } \\
\hline & & PSN & STA & $\mathrm{PF}$ & \\
\hline \multicolumn{6}{|l|}{ Chthamalus } \\
\hline PSN, STA & FA06 (35) & 27.2 & 0.876 & & 11.34 \\
\hline PSN, STA & FA07 (48) & 29.5 & 8.33 & & 11.28 \\
\hline PSN, STA & FA05 (23) & 12.2 & -3.34 & & 10.51 \\
\hline PSN, PF & SU05 (19) & 7.72 & & 33.5 & 14.82 \\
\hline PSN, PF & FA05 (23) & 12.2 & & 37.3 & 14.18 \\
\hline PSN, PF & WI05 (15) & -1.36 & & 19.7 & 13.04 \\
\hline STA, PF & SP04 (3) & & -28.5 & -90.4 & 17.83 \\
\hline STA, PF & SU05 (19) & & -11.4 & 33.5 & 11.02 \\
\hline STA, PF & FA03 (0) & & -28.3 & -74.9 & 10.03 \\
\hline \multicolumn{6}{|l|}{ Mytilus } \\
\hline PSN, STA & SP08 (52) & 45.6 & 14.4 & & 14.52 \\
\hline PSN, STA & FA07 (48) & 47.1 & 15.6 & & 14.29 \\
\hline PSN, STA & SP07 (40) & 41.9 & 11.2 & & 13.03 \\
\hline PSN, PF & FA05 (23) & -7.33 & & 25.52 & 17.62 \\
\hline PSN, PF & SU05 (19) & -22 & & 0.0955 & 13.82 \\
\hline PSN, PF & FA04 (11) & -37.4 & & -10.6 & 12.96 \\
\hline STA, PF & SP06 (28) & & -10.2 & 33.8 & 14.63 \\
\hline STA, PF & SP08 (52) & & 14.4 & 47.8 & 13.47 \\
\hline STA, PF & FA05 (23) & & -17.1 & 25.2 & 13.24 \\
\hline \multicolumn{6}{|l|}{ Endocladia } \\
\hline PSN, STA & SU05 (19) & 17 & -21.5 & & 24.99 \\
\hline PSN, STA & WI05 (15) & 15.5 & -18.8 & & 16.7 \\
\hline PSN, STA & FA05 (23) & 2.41 & -27 & & 12.29 \\
\hline PSN, PF & FA07 (48) & -30.3 & & 5.84 & 22.16 \\
\hline PSN, PF & SP07 (40) & -24.5 & & -14.9 & 15.83 \\
\hline PSN, PF & SP06 (28) & 0.104 & & -22.1 & 11.46 \\
\hline STA, PF & WI05 (15) & & -18.8 & 33.1 & 23.64 \\
\hline STA, PF & FA04 (11) & & -11.2 & 36.8 & 17.2 \\
\hline STA, PF & FA07 (48) & & -28.3 & 5.84 & 12.56 \\
\hline \multicolumn{6}{|l|}{ Silvetia } \\
\hline PSN, STA & FA06 (35) & -29.8 & 22.4 & & 18.24 \\
\hline PSN, STA & FA07 (48) & -22.7 & 22.7 & & 16.86 \\
\hline PSN, STA & SP08 (52) & -20.3 & 11.7 & & 10.75 \\
\hline PSN, PF & SP06 (28) & -27.5 & & 26.6 & 14.58 \\
\hline PSN, PF & FA06 (35) & -29.8 & & 28.6 & 14.36 \\
\hline PSN, PF & FA05 (23) & -23.3 & & 28.4 & 14.2 \\
\hline STA, PF & WI05 (15) & & -15.4 & 22.1 & 16.46 \\
\hline STA, PF & FA05 (23) & & -4.03 & 28.4 & 14.01 \\
\hline STA, PF & SP06 (28) & & 0.422 & 26.6 & 12.67 \\
\hline
\end{tabular}

tween Stairs and Point Fermin all occurred later in succession, with 23, 28, and 52 mo all contributing to over $40 \%$ of the variation in trajectories. At all 3 of these timesteps, recovery plots at Point Fermin consisted of more late successional species (more positive scores) than Stairs relative to the controls (Table 3). Thus, as with the Chthamalus assemblage, both recovery rates and trajectories displayed significant geographic variation in the Mytilus assemblage.
In the Endocladia assemblage, the PC1 axis explained $54.4 \%$ of the variation in community recovery trajectories. The components with the highest loadings and positive coefficients were bare rock and Mytilus californianus, whereas the species with the most negative coefficients were Endocladia, the red alga Mastocarpus spp, crustose brown algae, and the green alga Cladophora columbiana (Table 2). In this assemblage, the component scores of a recovering community transitioned from positive (rock-dominated) to more negative (communities dominated by Endocladia and Mastocarpus) over time. The MDS plot showed that all 3 sites had distinct recovery trajectories and that recovery trajectories differed by site $(\mathrm{R}=0.541 ; \mathrm{p}=0.001)$ and not by plot size ( $R=-0.266 ; p=0.999$ ) (Fig. 7). As with the Chthamalus assemblage, the intervals driving differences between Point Sierra Nevada and Stairs in the Endocladia assemblage all occurred in the year 2005, and these intervals explained over $50 \%$ of the variation in trajectories (Table 3). In each case, PC1 scores were much more negative at Stairs, indicating a stronger shift towards recovery relative to Point Sierra Nevada, which had more positive scores at these intervals indicating more bare rock and earlier successional species. The most important 2 intervals driving differences between Point Sierra Nevada and Point Fermin were later in succession (48 and $40 \mathrm{mo}$, respectively) (Table 3) which together explained nearly $40 \%$ of the variation. At these timesteps, Point Sierra Nevada had more negative PC1 scores, indicating a more fully-recovered community than Point Fermin. The 2 most important time intervals driving the differences in recovery trajectories between Stairs and Point Fermin were 11 and $15 \mathrm{mo}$, which contributed to over $40 \%$ of the variation and where Stairs had more negative PC1 values indicating that the communities there were more intact (Table 3)

In the Silvetia assemblage, the PC1 axis explained $45.5 \%$ of the community variation in recovery trajec- 
tories in the Silvetia assemblage. Silvetia, Mytilus, and Cladophora had the highest loadings and positive coefficients while bare rock was strongly negative (Table 2), indicating that a recovering plot would be expected to transition from more negative PC1 values to more positive ones over time. The recovery trajectories at Point Sierra Nevada were visually distinct from those at Stairs and Point Fermin, showing a significant effect of site $(\mathrm{R}=0.49 ; \mathrm{p}=0.001)$ but not disturbance size $(\mathrm{R}=0.236 ; \mathrm{p}=0.995)$ (Fig. 7). The most important intervals driving differences between Point Sierra Nevada and Stairs were later in succession ( 35 and $48 \mathrm{mo}$ ), and together, these intervals accounted for nearly $40 \%$ of the variation in trajectories (Table 3).

\section{DISCUSSION}

The capacity of communities to recover from disturbances is a key component of the functioning of natural ecosystems and is becoming an important aspect of effective management (Hughes et al. 2005). The dramatic variation I observed in recovery rates and trajectories shows that resilience is both an inherently spatial and species-specific quality, such that differences in recovery rates are best understood in both in the context of life history characteristics of the disturbed taxa and of biogeographic region where the disturbance has occurred. The result that the barnacle assemblage showed the fastest overall recovery is not surprising given its relatively short lifespan and that it is well understood that barnacles typically settle onto bare rock (e.g., Raimondi 1988), which is the most available substratum after a clearing disturbance in this system. This rapidity of recovery in barnacle-dominated communities has also been observed by Viejo (2009). Barnacles produce larvae that can disperse on the order of 10s to 100s of kilometers (Newman \& Abbott 1980, Shanks et al. 2003), increasing the likelihood of the arrival of new propagules to a given location. Of the dominant intertidal species in this study, Chthamalus is the shortest-lived; it can reach reproductive maturity on the order of months (Hines 1978), and is, thus capable of producing new recruits relatively quickly after a disturbance. This increased window of reproductive activity likely results in a much higher availability of propagules compared to the other species in this study. On geographic scales, this attribute likely contributes to the overall availability of propagules at a given time or location (Chapman \& Underwood 1998). The life history characteristics of Endocladia also favor shorter population turnover times and likely affected overall recovery rates for this assemblage. Endocladia is capable of recruiting into clearances through expansion via vegetative growth as well as through either the settlement of haploid or diploid spores originating from adult thalli. These multiple mechanisms of new space occupation by the dominant taxon could contribute to the relatively rapid recovery of the Endocladia assemblage. The timing of recovery found in this study (from 11 to $>52 \mathrm{mo}$ ) in the Endocladia assemblage is consistent with the findings of Foster et al. (2003) who observed that larger $(1 \times 2 \mathrm{~m})$ disturbances varied in recovery times from 1 to $>6$ yr. However, while I observed little among-site variation in the Endocladia assemblage recovery rates, Foster et al. (2003) found more geographic variation and generally slower recovery at their southernmost site, Diablo Canyon, than Point Sierra Nevada. Foster et al. (2003) found similar rates of recovery between fall- and spring-cleared plots in 3 of the 6 sites, faster recovery of spring-cleared plots at 2 of the sites (including Diablo Canyon), and faster recovery in fall-cleared plots at Point Sierra Nevada. The current study is analogous to fall-cleared plots; however because of the variable effect of season in Foster et al. (2003), it is difficult to determine how the season of clearing may have affected variation in recovery rates across sites in the current study.

The Mytilus assemblage had highly variable recovery rates that were directly related to disturbance size at all locations. It is well documented that adult mussels have the capacity to encroach into gaps in mussel beds (e.g. Paine \& Levin 1981) so smaller plots will experience this to a higher degree due to their higher perimeter to area ratio. The larger the disturbance in a mussel bed, the greater the reliance will be on recovery by larval input (Connell \& Slatyer 1977). Mussels, like barnacles, have propagules that are long-dispersing, but, unlike barnacles, they have specific habitat requirements for settlement such that they do not typically settle on bare rock but instead attach to the byssal threads of adult conspecifics or in some cases, turf algae (Menge et al. 1994). This means that for mussel recovery by recruitment, there must be both available larval propagules and suitable settlement sites as well as facilitation by conspecifics or others. Since these requirements may not align spatially or temporally (i.e. there might be available propagules but no available settlement sites or vice versa) and since mussels are more slowgrowing than barnacles, the slower recovery of mussels relative to barnacles is consistent with their life history strategy. Recovery via encroachment vs. re- 
cruitment was not directly quantified in this study, but encroachment was inferred to some degree in all experimentally disturbed plots at all sites (by the increasing presence of adult mussels at the perimeter of experimental disturbances).

The Silvetia assemblage displayed the most variable, as well as the slowest, recovery rates observed in this study (Figs. $2 \& 6$ ). Since the smallest recovery plots typically had a greater proportion of the plot covered by canopy from surrounding undisturbed individuals, small disturbances in this assemblage recovered very quickly, contributing to the overall variability in recovery rates in this assemblage. Larger disturbances were more likely to be reliant on successful propagule establishment for recovery. Silvetia releases thousands of gametes that have very high fertilization success but very low recruitment and subsequent colonization rates (Brawley \& Johnson 1991). Silvetia lives for several years (Gunnill 1980) so relatively few successful recruitment events are required for population persistence. Silvetia propagules (zygotes) disperse on the order of centimeters to a few meters (Brawley \& Johnson 1991). This means that the recovery from disturbances through successful recruitment events will be dependent on local fecundity and post-settlement survival, both of which may show considerable seasonal and geographic variability. In particular, benign microhabitats such as facilitator species (Brawley \& Johnson 1991) or topographic heterogeneity (Lubchenco 1983) have been shown to be important in increasing the likelihood of juvenile fucoid survival. Geographic or local variation in the availability of such microhabitats as well as in the importance of grazers (Coleman et al. 2006) are likely contributors to the extreme variation in recovery rates that I recorded for this assemblage and to the very slow recovery rates observed at Point Sierra Nevada. Previous studies of fucoid recovery have also showed variation and maximum recovery times on the order of years (e.g. Farrell 1991).

Both invertebrate-dominated assemblages (Mytilus and Chthamalus) showed spatial variation in recovery rates, with the slowest rates observed at Stairs, the site closest to Point Conception. This general pattern was observed by Raimondi et al. (1999) for zone-specific Mytilus and Chthamalus recruitment, and an investigation by Broitman et al. (2008) of mussel and barnacle recruitment in the mussel zone also showed a marked increase in Chthamalus recruitment south of Point Conception, while finding similar levels of Mytilus recruitment both north and south of the point. This suggests that geographically varying recruitment and/or larval supply, particularly for Chthamalus, are possible mechanisms driving the observed pattern of fastest recovery of Chthamalus south of Point Conception (Fig. 5), although this may not explain differences between the northern 2 sites. Higher growth rates of mussels in the warmer water south of Point Conception (Blanchette et al. 2007) as well as spatial variability in rates of predation (Menge et al. 2004) likely contribute to the geographic variability observed in recovery rates and trajectories of mussels, such that higher growth rates south of Point Conception may have compensated for similar rates of input. The contrasting rates of mussel recovery observed in this study between Point Sierra Nevada and Stairs differ from recovery observations made by Kinnetic Laboratories (1992) who found the opposite pattern in recovery rates for bigger $(1 \times 2 \mathrm{~m})$ clearings between Pt. Sierra Nevada and Diablo Canyon, a site north of Stairs, suggesting that the geographic patterns observed in this study may not be representative of each region as a whole.

I also found variable effects of disturbance size across study locations that differed with intertidal assemblage (Fig. 6). The manner in which sites vary in their response to different sizes of disturbance is suggestive of potentially different mechanisms at play across sites. For example, in the Chthamalus assemblage, the site with the slowest recovery rate (Stairs) was also the only site to show a pattern with respect to disturbance size. In this assemblage, gregariousness with conspecific adults (positive edge effects) appeared to be driving the increased recovery rates in smaller plot sizes. Thus, where recovery is faster, such edge effects may be less important, suggesting that the strength of interspecific interactions varies across locations, a phenomenon that has been documented in intertidal systems for trophic interactions, such as predation (Menge et al. 2004) and herbivory (Coleman et al. 2006). Alternatively, edge effects may be similar at the 3 sites, but where recovery occurs faster, such effects may have required more frequent observations to be measured effectively.

Strikingly, in the Endocladia assemblage, the edge had a trend towards positive effects at 2 sites (Point Sierra Nevada and Point Fermin) but had a significant negative effect at Stairs. This result is consistent with the findings of Sousa (1984) who found greater abundances of Endocladia in larger experimental patches than in smaller ones, indicating that the center of large disturbances at this site could be a refuge from herbivores or other negative edge effects such 
as shading. It also challenges the notion that the relationship between recovery rate and disturbance size is static for a given species or location and underscores the importance of conducting studies across broad spatial scales.

The timing of key events driving differences in community recovery trajectory (Fig. 7) differed across sites and assemblages (Table 3). In some cases, such as between the Chthamalus plots at Point Sierra Nevada and Point Fermin, the important intervals identified were relatively close to one another in time, suggesting a major series of ecologically relevant occurrences (i.e. recruitment, predation, or growth events) as being important in the divergences between pairs of sites (Table 3). In this case, since Point Fermin showed positive principal component scores relative to Point Sierra Nevada at all 3 intervals (Table 3), an increase in Chthamalus cover driven by recruitment or growth was likely responsible. By contrast, other comparisons identified intervals, both early and in late succession, that drove differences in recovery trajectories (e.g. Endocladia plots at Stairs and Point Fermin) and suggested events that led to higher cover of Endocladia and Mastocarpus at Stairs (more negative PC values) than at Point Fermin (Table 3) throughout succession. The extent to which recovery rates and trajectories mirror one another can also provide important insights into the recovery process. I found that successional trajectories were different across geographic locations within all 4 intertidal assemblages and appeared to be driven by differences in the timing of community shifts from bare rock and early-mid successional species to a greater abundance of the dominant species in each assemblage (Tables $2 \& 3$ ). I found these differences even in the 2 algal-dominated intertidal assemblages where community recovery rates were statistically similar, suggesting that similar recovery rates may be driven by very different ecological processes. Since, like most species of algae, both Endocladia and Silvetia have propagules that disperse at more local scales (meters rather than kilometers), the timing and availability of viable recruits is likely to be reliant to some degree on local fecundity, which can differ seasonally and geographically (Foster et al. 2003) and thus contribute to observed differences in the timing of community shifts towards later successional taxa. Spatial and temporal variation in key processes, such as recruitment and consumer pressure, combined with site-specific differences in the relative importance of mechanisms driving recovery may also contribute to observed geographic variation in both recovery rates and trajectories.

\section{CONCLUSIONS}

As both ecological insights (e.g. Gouhier et al. 2010) and resource management (Halpern et al. 2007) shift toward more spatially explicit approaches, understanding variation in the likely responses to natural and anthropogenic perturbations is becoming increasingly important. Here, I have demonstrated that, for disturbances that result in complete loss of biomass (e.g, rock breaking, pressure-washed oil spills), shorter-lived taxa are more likely to show faster recovery, and thus higher resilience, than those that are longer-lived, and that the 2 longer-dispersing taxa in this study showed similar geographic variation in recovery rates. Importantly, I show that while there exist some generalities across locations in recovery rates, recovery trajectories were distinct for each location, highlighting the need for elucidation of both site-specific mechanisms of recovery and the need to consider variation in recovery in management planning.

Acknowledgements. I am deeply grateful to P. Raimondi, D. Doak, S. Murray, I. Parker, and J. Pearse for their invaluable insights and editorial comments throughout this study. I am also grateful for the thoughtful and thorough comments of 3 anonymous reviewers. This work could not have been completed without the tireless assistance in the field from many people including M. Miner, C. Bell, H. Hayford, M. Bond, P. Raimondi, D. Lohse, L. Anderson, L. Reeve, H. Livingston, A. Kendall, M. Williams and M. Helix. For access to field sites and logistical help I thank J. Milgrim, K. Milgrim, D. Canestro, M. Canestro, the Hearst Ranch Corporation, Vandenberg Air Force Base, Rancho Marino University of California Reserve and the Cabrillo Aquarium. This paper benefited from discussions and comments from T. Klinger. Funding for this study was provided by the Mineral Management Service (MMS), the Partnership for Interdisciplinary Studies of Coastal Oceans (PISCO), a National Science Foundation dissertation improvement grant (DEB 0808012), Friends of Long Marine Lab, the Earl and Ethel Meyers Oceanographic Trust, and the Marilyn C. Davis Memorial Grant.

\section{LITERATURE CITED}

Allison G (2004) The influence of species diversity and stress intensity on community resistance and resilience. Ecol Monogr 74:117-134

Bazzaz FA (1979) Physiological ecology of plant succession. Annu Rev Ecol Syst 10:351-371

Bertness MD, Shumway SW (1993) Competition and facilitation in marsh plants. Am Nat 142:718-724

Bertness MD, Trussell GC, Ewanchuk PJ, Silliman BR (2002) Do alternate stable community states exist in the Gulf of Maine rocky intertidal zone? Ecology 83:3434-3448

> Blanchette CA, Helmuth B, Gaines SD (2007) Spatial patterns of growth in the mussel, Mytilus californianus, across a major oceanographic and biogeographic bound- 
ary at Point Conception, California, USA. J Exp Mar Biol Ecol 340:126-148

Blanchette CA, Miner CM, Raimondi PT, Lohse D, Heady KEK, Broitman BR (2008) Biogeographical patterns of rocky intertidal communities along the Pacific coast of North America. J Biogeogr 35:1593-1607

Brawley SH, Johnson LE (1991) Survival of fucoid embryos in the intertidal zone depends upon developmental stage and microhabitat. J Phycol 27:179-186

$>$ Bray JR, Curtis JT (1957) An ordination of the upland forest communities of southern Wisconsin. Ecol Monogr 27: 325-349

> Broitman BR, Blanchette CA, Menge BA, Lubchenco J and others (2008) Spatial and temporal patterns of invertebrate recruitment along the west coast of the United States. Ecol Monogr 78:403-421

Burrows MT, Harvey R, Robb L, Poloczanska ES and others (2009) Spatial scales of variance in abundance of intertidal species: effects of region, dispersal mode, and trophic level. Ecology 90:1242-1254

> Cervin G, Lindegarth M, Viejo RM, Aberg P (2004) Effects of small-scale disturbances of canopy and grazing on intertidal assemblages on the Swedish west coast. J Exp Mar Biol Ecol 302:35-49

> Chapman MG, Underwood AJ (1998) Inconsistency and variation in the development of rocky intertidal algal assemblages. J Exp Mar Biol Ecol 224:265-289

> Clarke KR, Somerfield PJ, Airoldi L, Warwick RM (2006) Exploring interactions by second-stage community analyses. J Exp Mar Biol Ecol 338:179-192

> Coleman RA, Underwood AJ, Benedetti-Cecchi L, Aberg P and others (2006) A continental scale evaluation of the role of limpet grazing on rocky shores. Oecologia 147: 556-564

Connell JH, Slatyer RO (1977) Mechanisms of succession in natural communities and their role in community stability and organization. Am Nat 111:1119-1144

> Connell JH, Sousa WP (1983) On the evidence needed to judge ecological stability or persistence. Am Nat 121: 789-824

> Connolly SR, Menge BA, Roughgarden J (2001) A latitudinal gradient in recruitment of intertidal invertebrates in the northeast Pacific Ocean. Ecology 82:1799-1813

Crain CM, Albertson LK, Bertness MD (2008) Secondary succession dynamics in estuarine marshes across landscape-scale salinity gradients. Ecology 89:2889-2899

Cudaback CN, Washburn L, Dever E (2005) Subtidal innershelf circulation near Point Conception, California. J Geophys Res 110:C10007 doi: 10.1029/2004JC002608

> Dietze MC, Clark JS (2008) Changing the gap dynamics paradigm: vegetative regeneration control on forest response to disturbance. Ecol Monogr 78:331-347

> Dudgeon S, Petraitis PS (2001) Scale-dependent recruitment and divergence of intertidal communities. Ecology 82: 991-1006

> Elmqvist T, Folke C, Nystrom M, Peterson G, Bengtsson J, Walker B, Norberg J (2003) Response diversity, ecosystem change, and resilience. Front Ecol Environ 1: 488-494

> Farrell TM (1989) Succession in a rocky intertidal community: the importance of disturbance size and position within a disturbed patch. J Exp Mar Biol Ecol 128:57-73

Farrell TM (1991) Models and mechanisms of succession: an example from a rocky intertidal community. Ecol Monogr 61:95-113
Foster MS, Nigg EW, Kiguchi LM, Hardin DD, Pearse JS (2003) Temporal variation and succession in an algaldominated high intertidal assemblage. J Exp Mar Biol Ecol 289:15-39

> Gouhier TC, Guichard F, Menge BA (2010) Ecological processes can synchronize marine population dynamics over continental scales. Proc Natl Acad Sci USA 107: 8281-8286

> Gunnill FC (1980) Demography of the intertidal brown alga Pelvetia fastigiata in southern California, USA. Mar Biol 59:169-179

Haderlie EC, Abbott DP (1980) Bivalvia: the clams and allies. In: Morris RJ, Abbott DP, Haderlie EC (eds) Intertidal invertebrates of California. Stanford University Press, Stanford, CA

Halpern BS, Selkoe KA, Micheli F, Kappel CV (2007) Evaluating and ranking the vulnerability of global marine ecosystems to anthropogenic threats. Conserv Biol 21: 1301-1315

Hays C (2006) Ecological consequences of dispersal and gene flow in an intertidal alga. PhD dissertation, University of California, Santa Cruz, CA

- Hickey BM (1979) The California current system - hypotheses and facts. Prog Oceanogr 8:191-279

Hickey BM (1993) Physical oceanography. In: Dailey MD, Reish DJ, Anderson JW (eds) Ecology of the Southern California Bight. University of California Press, Berkeley, CA p $19-70$

Hines AH (1978) Reproduction in three species intertidal barnacles from central California. Biol Bull 154:262-281

Holling CS (1973) Resilience and stability of ecological systems. Annu Rev Ecol Syst 4:1-23

> Hubbell SP, Foster RB, O'Brien ST, Harms KE and others (1999) Light-gap disturbances, recruitment limitation, and tree diversity in a neotropical forest. Science 283: $554-557$

> Hughes TP, Bellwood DR, Folke C, Steneck RS, Wilson J (2005) New paradigms for supporting the resilience of marine ecosystems. Trends Ecol Evol 20:380-386

Jenkins SR, Hawkins SJ, NortonTA (1999) Interaction between a fucoid canopy and limpet grazing in structuring a low shore intertidal community. J Exp Mar Biol Ecol 233:4-63

Kinnetic Laboratories (1992) Study of the rocky intertidal communities of central and northern California, Vol. 1 OCS Study MMS 91-0089. US Department of Interior, Los Angeles, CA

> Lebrija-Trejos E, Perez-Garcia EA, Meave JA, Bongers F, Poorter L (2010) Functional traits and environmental filtering drive community assembly in a species-rich tropical system. Ecology 91:386-398

Leslie H, Kingzig A (2007) Resilience science. In: Leslie HaKM (ed) Ecosystem-based management for the oceans. Island Press, Washington, DC

> Lubchenco J (1983) Littornia and Fucus: Effects of herbivores, substratum heterogeneity, and plant escapes during succession. Ecology 64:1116-1123

> Lubchenco J, Menge BA (1978) Community development and persistence in a low rocky intertidal zone. Ecol Monogr 48:67-94

Menge BA, Branch GM (2001) Rocky intertidal communities. In: Bertness MD, Gaines SD, Hay ME (eds) Mar Comm Ecol. Sinauer Associates, Sunderland, MA p 221-251

Menge BA, Berlow EL, Blanchette CA, Navarrete SA, 
Yamada SB (1994) The keystone species concept: variation in interaction strength in a rocky intertidal habitat. Ecol Monogr 64:249-286

Menge BA, Blanchette C, Raimondi P, Freidenburg T and others (2004) Species interaction strength: testing model predictions along an upwelling gradient. Ecol Monogr 74:663-684

Murray SN, Littler MM (1978) Patterns of algal succession in a perturbated marine intertidal community. J Phycol 14: 506-512

Newman WA, Abbott DP (1980) Cirripedia: the barnacles. In: Morris RJ, Abbott DP, Haderlie EC (eds) Intertidal invertebrates of California. Stanford University Press, Stanford, CA

O'Leary JK, McClanahan TR (2010) Trophic cascades result in large-scale coralline algae loss through differential grazer effects. Ecology 91:3584-3597

Paine RT (1966) Food web complexity and species diversity. Am Nat 100:65-75

Paine RT, Levin SA (1981) Intertidal landscapes: disturbance and the dynamics of pattern. Ecol Monogr 51:145-178

Petraitis PS, Dudgeon SR (1999) Experimental evidence for the origin of alternative communities on rocky intertidal shores. Oikos 84:239-245

Petraitis PS, Dudgeon SR (2005) Divergent succession and implications for alternative states on rocky intertidal shores. J Exp Mar Biol Ecol 326:14-26

Phillips DL, Shure DJ (1990) Patch-size effects on early succession in southern Appalachian forests. Ecology 71: 204-212

Raimondi PT (1988) Rock type affects, settlement, recruitment and zonation of the barnacle Chthamalus anisopoma Pilsbury. J Exp Mar Biol Ecol 123:253-267

Editorial responsibility: Steven Morgan, Bodega Bay, California, USA
Raimondi PT (1990) Patterns, mechanisms, consequences of variability in settlement and recruitment of an intertidal barnacle. Ecol Monogr 60:283-309

Raimondi PT, Engle J, Ambrose RA, Murray SN, Wilson M, Sapper S (1999) Rocky Intertidal resources in San Luis Obispo, Santa Barbara, and Orange Counties, 3 year report, Minerals Management Service, Camarillo, CA

Shanks AL, Grantham BA, Carr MH (2003) Propagule dispersal distance and the size and spacing of marine reserves. Ecol Appl 13:159-169

Sousa WP (1979) Experimental investigations of disturbance and ecological succession in a rocky intertidal agal community. Ecol Monogr 49:227-254

> Sousa WP (1984) Intertidal mosaics: patch size, propagule availability, and spatially-variable patterns of succession. Ecology 65:1918-1935

> Suchanek TH (1981) The role of disturbance in the evolution of life history strategies in the intertidal mussels Mytilus edulis and Mytilus californianus. Oecologia 50:143-152

Tilman D (1985) The resource-ratio hypothesis of plant succession. Am Nat 125:827-852

> Underwood AJ (2000) Experimental ecology of rocky intertidal habitats: What are we learning? J Exp Mar Biol Ecol 250:51-76

> van Nes EH, Scheffer M (2007) Slow recovery from perturbations as a generic indicator of a nearby catastrophic shift. Am Nat 169:738-747

> Viejo RM (2009) Resilience in intertidal rocky shore assemblages across the stress gradient created by emersion times: Mar Ecol Prog Ser 390:55-65

Young CM, Gotelli NJ (1988) Larval predation by barnacles - effects on patch colonization in a shallow subtidal community. Ecology 69:624-634

Submitted: September 11, 2011; Accepted: March 11, 2012 Proofs received from author(s): June 4, 2012 